\title{
Review of Voice Controlled Robotic Arm-Raspberry Pi
}

\author{
B. Shoban Babu, V. Priyadharshini, and Prince Patel
}

\begin{abstract}
One of the most essential life skills is to be able to communicate easily. In order to produce greater comprehension, communication is described as transmitting knowledge. Communication and technologies are not mutually exclusive. Speech Recognition is a technique that facilitates the processing of voice information to text and is independent of the speaker. This enables it to be used in various applications, from digital assistants to machinery control. The aim of this paper is to study numerous robotic vehicles powered by human speech commands. To accomplish this functionality, most of these systems run with the use of an android smart phone that transmits voice commands to a raspberry pi. The voice-operated robot is used to build one moving object. It is moved as per the voice recognition module commands, and the robot obtains that command. The robot compares the command with the stored software and then sets the command using wireless communication as per voice. These suggested methods would be useful for devices such as assistive robotics for people with disabilities or automotive applications such as work robots.
\end{abstract}

Index Terms - Robotics, LeenaBOT, Raspberry Pi.

\section{INTRODUCTION}

Robots are a package of systems of technology which can be used to perform diverse tasks in industrial and domestic use. With growing advances in the field of robotics, robots can now be controlled to create a more natural interface with machines with less overt human interference.

Some basic applications of robots utilizing voice recognition are to support elderly people or people with disability, voice controlled personal assistance etc [1], [2]. The Raspberry pi board has several applications, from distance measurement of a target object from another object for the purpose of civil engineering [3] to developing a robotic arm to help medical staff and elderly people. Instead of using multiple dynamic interfaces, it is easier to communicate with machines through voice commands.

Bluetooth technology communicates knowledge over a short range, but it is a very capable way to connect between two devices, such as a microcontroller and a mobile phone [4]. Packages of data are transmitted and received via shortwave radio signals. The speech recognition system is used as the user interface for running the robot in the models shown. Firstly, via android smart phone, we have to send voice commands. These commands are processed in the software of smart phones and are then transmitted to Bluetooth modem which is linked to the Raspberry Pi board [5]. The Raspberry pi processes these commands and in turn

Submitted on February 02, 2020.

Published on March 10, 2021.

Dr. B. Shoban Babu, SV College of Engineering, Tirupati, India. (e-mail: bshobanbabu@gmail.com)

V. Priyadharshini, Sona College of Technology, Anna University, Coimbatore, India.

(e-mail: priya.vairamanickam ${ }^{\circledR}$ gmail.com) the robot behaves as commanded. With the support of the two basic features, speech recognition and Bluetooth connectivity, the robot can be used commercially and domestically for variable purposes and applications. To increase the performance of such robots, it is important to make more technical advancements in voice recognition systems.

This research aims to define and present the build and working of Raspberry Pi projects and the abilities to develop robots that can be controlled via voice command. The remainder of this article is structured as follows. The necessary information about Raspberry $\mathrm{Pi}$ is presented in Section 2. Voice Controlled Robot build and working, in Section 3. Finally, concluding remarks are given in Section 4.

\section{RASPBERRY PI}

Raspberry $\mathrm{Pi}$ is a series of small single-board computers developed in the United Kingdom by the Raspberry Pi Foundation in association with Broadcom. The Raspberry Pi is a lightweight, powerful, inexpensive, hackable, and educational computer board [6] (Fig. 1). The Raspberry Pi was introduced in 2012, and several versions and variants have since been released. The original Raspberry Pi had a single-core $700 \mathrm{MHz} \mathrm{CPU}$ and just 256MB RAM, and a quad-core $1.4 \mathrm{GHz}$ CPU with $1 \mathrm{~GB}$ RAM is available for the new edition. For Raspberry Pi, the main price point has always been $\$ 35$, and all models have been $\$ 35$ or less, including the Pi Zero, which is just $\$ 5$ [7] (Fig. 1). Some of these devices are necessary, others are optional, but all Raspberry Pi models have the same BCM2835 CPU, which is inexpensive, efficient, and does not consume a lot of energy. The Raspberry Pi also utilizes an operating system like any other computer.

In order to learn programming skills, create hardware projects, do home automation and even use them in industrial applications, people all over the world use Raspberry Pi. There have been three generations of Raspberry Pi`s: Pi 1, Pi 2, and Pi 3, and most generations have usually had a Model A and a Model B. Model A is a cheaper version which appears to have lower RAM and USB and Ethernet ports. The Pi Zero is a spinoff, made much smaller and cheaper, of the original (Pi 1) generation. Table I provides a comparison of the scale, weight, and cost of the standard Raspberry Pi generation.

Prince Patel, Student, Charotar University of Science and Technology Gujarat, India

(e-mail: princepate1221375@gmail.com) 


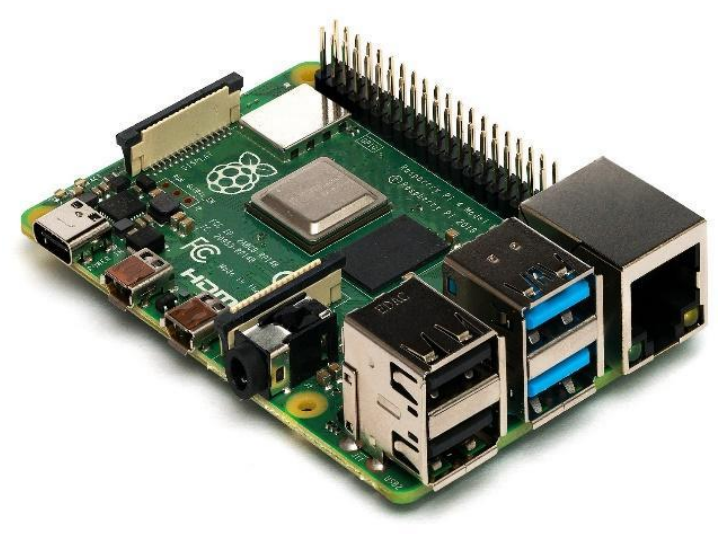

Fig. 1. Raspberry Pi 4 Model B.

The values given in Table 1 illustrate that Raspberry Pi's superiority over other systems lies in its lowest price per device.

\begin{tabular}{cccc}
\multicolumn{4}{c}{ TABLE I: THE COMPARISON OF Size, WEIGHT, AND COST } \\
\hline \multirow{2}{*}{ Name } & $\begin{array}{c}\text { Size } \\
(\mathrm{mm})\end{array}$ & $\begin{array}{c}\text { Weight } \\
(\text { gram })\end{array}$ & $\begin{array}{c}\text { Cost } \\
(\text { US \$) }\end{array}$ \\
\hline Pi 2 & $56.5^{*} 85.6 * 17$ & 45 & 35 \\
Pi 3 & $85.6 * 56.5 * 17$ & 45 & 35 \\
Pi 4 B & $56.5^{*} 85.6 * 11$ & 46 & 35 \\
\hline
\end{tabular}

\section{PROTOTYPE}

In this section, we study two different Raspberry Pi prototype projects that use voice control in some way or another. We take a look at the build of the prototypes and understand the working of the prototype.

A robotic arm is a type of mechanical arm that is typically programmable and has similar skills to a human arm. The most significant advantage that robotic assistance provides is working in a hazardous area, or it can be used when an actual human is unavailable at the desired location. A voicecontrolled robotic arm is simple and helps the user control the robot with a high level of abstraction from the robot, making it seamless to operate at times.

Senior citizens and physically challenged people will need assistants most of the time, even for small tasks like getting water, food, and clothes. To assist senior citizens and the physically challenged, a robotic assistant car controlled with a voice command using Raspberry pi can come in handy [2], [7]. A robotic arm using a Bluetooth connection between a phone and raspberry pi can be controlled through a voice command feature. Via the Android phone, we deliver an input voice [8], [9]. Digital cameras, the Android operating system typically used for cell phones, are user-friendly and can be operated by anyone. A Bluetooth module is paired with an Android phone and a robotic arm. Hand movements are conducted using servo motor circuits that are positioned and aligned at particular rotations and positions. The regulation of movement is achieved by a microcontroller. A voice command is obtained from the smartphone by the controller and the nature of the movement depends on the voice commands received. Fig. 2 shows the flow chart diagram of the system.

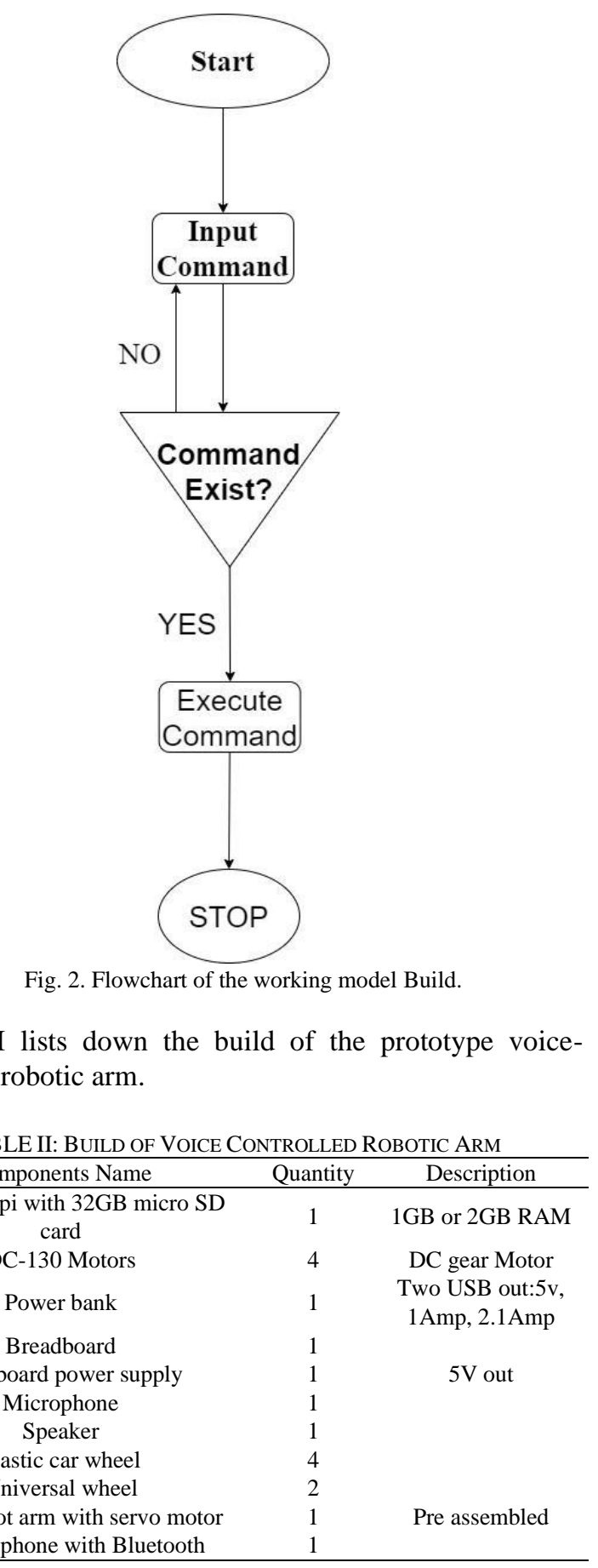

\section{A. Power Supply}

This segment is concerned with the robot's power requirements for DC motors. An essential need for designing any device is the power supply. The Raspberry $\mathrm{Pi}$ is powered by a $+5 \mathrm{v}$ micro-USB power supply and the exact current needed depends on the connections to the Raspberry Pi and the $5 \mathrm{v}$ power bank is used for the power supply [10]. 


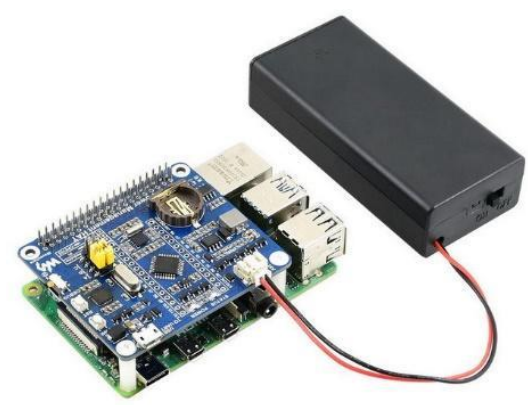

Fig. 3. Power bank.

\section{B. Raspberry Pi}

The Raspberry Pi 4 model B is used for the proposed prototype. It features a Broadcom system on a chip which consists of ARM-compatible A-72 CPU and on-chip Graphics Processing Unit GPU and 32 GB micro-SD card for the memory. It also has Wi-Fi and Bluetooth port and used Bluetooth 5.0. Python is main programming language where C, C++, PHP, Java and more also available and can cost from anywhere around 35 \$ to 45 \$ US. Other model of the Raspberry can be used but for the proposed prototype Raspberry Pi B board is being used.

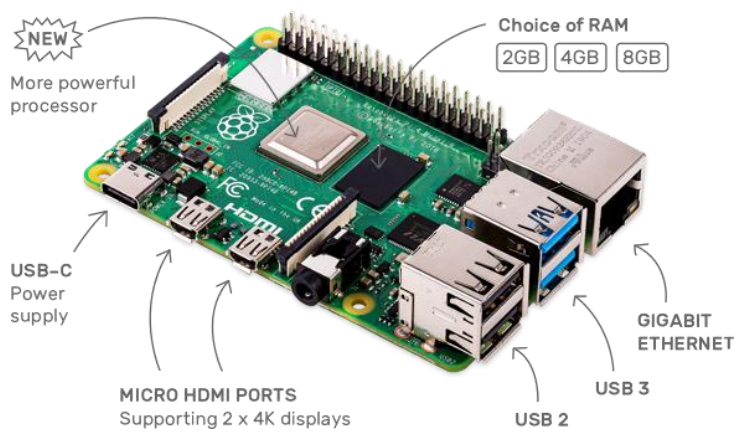

Fig. 4. Raspberry Pi 4 B.

\section{Bluetooth Module}

Bluetooth is a wireless technology that uses a short-range wireless link to easily connect cell phones, PDAs, and personal computers to each other [11]. Wireless Bluetooth communication can be formed between two devices using MAC addresses using a Bluetooth module chip, as each device has its own unique MAC address. The robot car with the robot arm is connected to Raspberry Pi 4. The Raspberry pi 4 has inbuilt Bluetooth version 5.0. By writing a Bluetooth server program at raspberry pi, we will always listen for incoming connections.

\section{Servo Motor}

A servo motor is a type of motor which can rotate very accurately. This type of motor usually consists of a control circuit that provides feedback on the current location of the motor shaft, allowing the servo motors to rotate with great precision. The servo engine produces $1.8 \mathrm{Kg} . \mathrm{cm}$ of slow down torque at $4.8 \mathrm{~V}$ and $2.2 \mathrm{Kg} . \mathrm{cm}$ at $6 \mathrm{~V}$ [12]. For rakish movement, servo engines are used, and we can know precisely the edge at which the engine is turned at a particular stage. From the focal start point, most servo engines turn $90^{\circ}$ on either side on a regular basis [13].

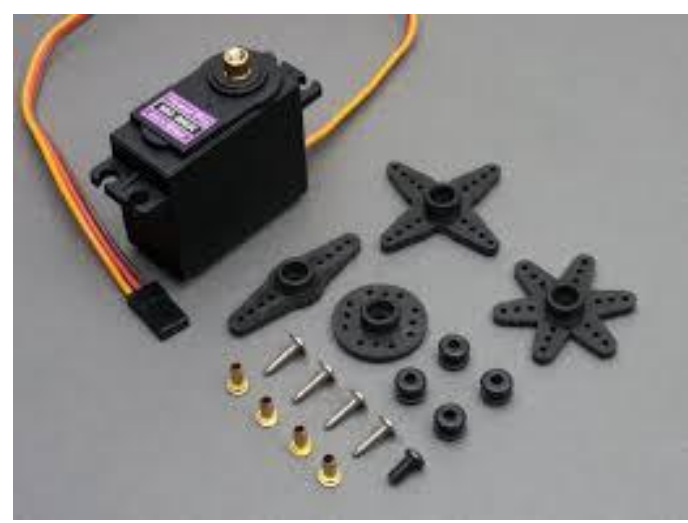

Fig. 5. Servo Motor MG996.

\section{E. System Interface}

Using a smartphone will send our voice command. Fig. 6 depicts the system diagram of the robotic arm.
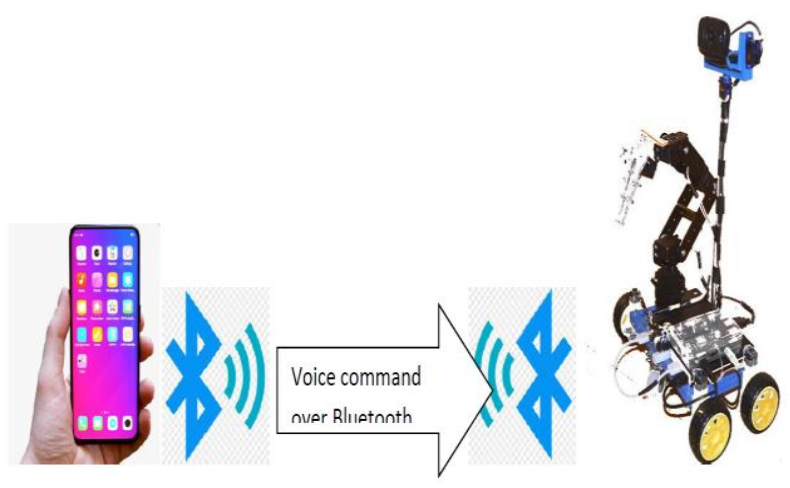

Fig. 6. System Interface Diagram.

\section{F. Software}

The design implements two software modules. The first module is server-side python code to receive data from the phone. The second module is a client-side application installed on the phone to send voice commands to the robot arm. Server-side python code combines Bluetooth socket program and driver code to drive servo motor and DC-130 motors. Server code waits for incoming connections [14]. Once the connection is established its waits for the control voice commands and sends signals to each motor according to the voice command received. When connected to raspberry pi, the Bluetooth application can send an order to the robotic arm. The robotic arm acts following the voice command it receives via the application.

\section{FUTURE SCOPE AND CONCLUSION}

Future work will focus on presenting the framework with increasingly complicated activities and sentences. To increase current human capacities, the same technologies can be used. In conditions that are harmful to humans, such as industrial welding, disaster response, etc., robotic arms may be used [15]. Today, imagination is developing a similar solution in line with rapidly increasing human needs. 


\section{REFERENCES}

[1] Prakash Kanade, Sunay Kanade, "Medical Assistant Robot ARM for COVID-19 Patients Treatment - A Raspberry Pi Project," International Research Journal of Engineering and Technology (IRJET), vol. 7, no. 10, Pages. 105-111, 2020.

[2] Prakash Kanade, Sunay Kanade, "Raspberry Pi Project - Voice Controlled Robotic Assistant for Senior Citizens," International Research Journal of Engineering and Technology (IRJET), vol. 07, no. 10, Pages. 1044-1049, 2020.

[3] Prakash Kanade, Prajna Alva, "RASPBERRY PI PROJECT ULTRASONIC DISTANCE SENSOR IN CIVIL ENGINEERING," International Journal in IT \& Engineering (IJITE), vol. 8, no. 10, Pages. 1-6, 2020.

[4] M. Ryan, "Bluetooth: With low energy comes low security," Workshop on Offensive Technologies, vol. 13, 2013.

[5] V. a. M. M. Vujović, "Raspberry Pi as a Wireless Sensor node: Performances and constraints," 37th International Convention on Information and Communication Technology, Electronics and Microelectronics (MIPRO). IEEE, 2014.

[6] M. V. V. N. D. V. M. a. B. P. Maksimović, "Raspberry Pi as Internet of things hardware: performances and constraints," Proceedings of 1 st International Conference on Electrical, Electronic and Computing Engineering, 2014.

[7] Prakash Kanade, Monis Akhtar, Fortune David, "Computer Networking and Technology Improvement in the Age of COVID19," International Journal of Advanced Networking and Applications (IJANA), vol. 12, no. 03, Pages. 4592-4595, 2020.

[8] M. M. S. a. R. S. Vanitha, "Monitoring and controlling of mobile robot via internet through raspberry Pi board," Second International Conference on Science Technology Engineering and Management (ICONSTEM). IEEE, 2016.

[9] R. V. P. A. d. O. B. S. M. L. \&. G. G. Aroca, "Towards smarter robots with smartphones," 5th workshop in applied robotics and automation, Robocontrol , pp. 1-6, 2012.

[10] S. U. UPase, "Speech recognition based robotic system of wheelchair for disable people," 2016 International Conference on Communication and Electronics Systems (ICCES). IEEE, 2016.

[11] R. A. a. R. Y. Rashid, "Bluetooth performance analysis in personal area network (PAN)," 2006 International RF and Microwave Conference. IEEE, 2006.

[12] N. S. J. S. M. T. S. S. a. R. K. C. Andrews, "Low-Cost Robotic Arm for differently abled using Voice Recognition," 3rd International Conference on Trends in Electronics and Informatics (ICOEI), pp. 735-739, 2019.

[13] Prakash Kanade, Prajna Alva, Sunay Kanade, Shama Ghatwal, "Automated Robot ARM using Ultrasonic Sensor in Assembly Line," International Research Journal of Engineering and Technology (IRJET), vol. 07, no. 12, Pages. 615-620, 2020.

[14] Prakash Kanade, Monis Akhtar, Fortune David, "Remote Monitoring Technology for COVID-19 Patients," European Journal of Electrical Engineering and Computer Science, vol. 5, no. 1, Pages. 44-47, 2021.

[15] Prakash Kanade, Ashwini P, "Smart Agriculture Robot for Sowing Seed," International Journal of Engineering Science and Computing (IJESC), vol. 11, no. 01, Pages. 27563-27565, 2021. 